\title{
The myth about consent forms
}

\section{Russell Heathcote-Curtis}

Dento-legal Advisor, BDA Indemnity

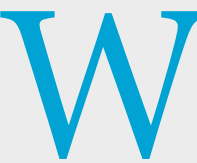

hen dental professionals are trained, consent is usually taught as part of a law and ethics curriculum. The

background of the personnel providing this training will largely determine just where the emphasis is placed in this potentially complex subject. Sometimes the legal aspects of consent will dominate discussions, whilst on other occasions the teaching may be left to someone with no formal legal training, and here the emphasis might have a more practical clinical focus and, by comparison, it might appear to be more simplistic in nature.

At both extremes, but for different reasons, the process of obtaining consent can soon appear to be predominantly concerned with getting the patient's signature on a form to protecting an individual or an institution. But It is a complete myth that the patient's signature at the end of the form provides such protection. The origin of this misunderstanding has been lost in the mists of time, but that doesn't alter the fact that asking the patient to sign a form does little other than confirm that they signed the form on the date next to their signature.

The reason for this is simply that a signature on a 'consent form' is not, in itself, a reliable indication that the patient has actually understood any of the issues involved in the treatment they will be having - even if the form states that they have - or that the patient has given valid consent for a procedure.

The consent process is not about creating legal protection for the clinical team that will be providing treatment. It is a communication process that reflects the autonomy of the patient who will be receiving that treatment. Any consent form that you have asked the patient to sign, should be regarded as just one small part of the overall record of the communication that has taken place between patient and clinician in advance of treatment being provided. The larger part of the record needs to capture the essence of the information provided to the patient, the issues raised by any of the treatment options and the clinician's response along with the patient's final decision about their preferred choice from the options discussed.

Only in this way can it be demonstrated that the consent process was valid, should the question be raised at a later date. In spite of the long-standing myth, a signed form on its own does not demonstrate that valid consent had been obtained. https://doi.org/10.1038/s41404-020-0355-2

\section{Myths about needlesticks}

\section{Russell Heathcote-Curtis}

Dento-legal Advisor, BDA Indemnity

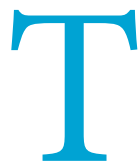
here is a confidence that comes from adopting the highest levels of infection control procedures in the dental setting. Whenever the dental team is asked to treat a new patient they can do so without any exaggerated sense of fear if the patient's history suggests they could have been exposed to HIV or Hepatitis.

But accidents do happen and the reality of a needlestick injury can be quite upsetting for the staff involved. And it could happen to any member of the dental team. The risk comes from needles and equipment that have been contaminated with the patient's blood and saliva, both whilst treatment is on-going and whilst clearing away after the patient has left and again during the decontamination cycle for reusable items.
The Health and Safety (Sharp Instruments in Healthcare) Regulations 2013 were introduced to help tackle the problem by requiring practice owners and supervising clinicians to undertake a risk assessment of the use of needles and other sharp items in the clinical setting and to introduce appropriate control measures. Amongst other things, those include:

$\rightarrow$ A written practice policy/protocol in place, including a risk assessment with justification for the continued use of reusable local anaesthetic syringes

$\rightarrow$ Only using sharps when required

$\rightarrow$ Substitution of 'safer sharps' where possible

$\rightarrow$ Secure sharps disposal containers close to the work area (not on the floor)

$\rightarrow$ Employee training to work safely with sharps they will use.

Staff must tell their employer about any sharps-related injuries in the surgery so that the cause of the incident can be investigated and any necessary action taken. If that person could have been exposed to a blood-borne

\section{Useful resources online}

\author{
$\rightarrow$ Health and Safety (Sharp \\ Instruments in Healthcare) \\ Regulations 2013 \\ $\rightarrow$ HSE guidelines \\ $\rightarrow$ European Council Directive \\ 2010/32/EU (the Sharps Directive) \\ $\rightarrow$ HTM 01-05: Decontamination in \\ primary care dental practices
}

virus, they will need swift access to medical advice which might involve post-exposure prophylaxis and possibly counselling. By finding the location and contact details of the nearest occupational health service or A and E Department and planning the quickest way to get there, could save a lot of anxiety for any member of the practice who finds themselves in this situation.

It goes without saying that a record should also be made in the practice accident and injury book. *

https://doi.org/10.1038/s41404-020-0368-x 